\title{
Synthesis, growth, and characterization of hexagonal shaped potassium-boro-phthalate (KBP) single crystal by slow solvent evaporation method for optoelectronics applications
}

\author{
K. Mohanraj, D. Balasubramanian*, N. Jhansi \\ Raman Research Laboratory, PG \& Research Department of Physics, Government Arts College, Tiruvannamalai-606603, \\ Tamilnadu, India
}

\begin{abstract}
A novel nonlinear optical crystal of potassium-boro-phthalate (KBP) was grown by a slow solvent evaporation technique. The crystalline nature and cell parameters were analyzed using X-ray diffraction (XRD) study. The presence of various functional groups was determined by Fourier transform infrared spectroscopy (FT-IR) spectral analysis. The linear optical properties were determined by UV-Vis-NIR spectral analysis. The dielectric constant and dielectric loss of KBP were measured in the frequency range of $500 \mathrm{~Hz}$ to $5 \mathrm{MHz}$ at different temperatures. The mechanical properties of KBP single crystal were studied using Vickers microhardness tester. The linear and nonlinear optical properties of the grown crystals were studied to assess its suitability for device fabrication.
\end{abstract}

Keywords: dielectric materials; microhardness; second harmonic

\section{Introduction}

Nonlinear optical (NLO) crystals contain various types of bonds, including ionic (cationanion), covalent (intermolecular), Van der Walls and hydrogen bonds [1-3]. The NLO behavior of the crystal is due to the influence of strong and weak hydrogen bonds as already reported [4]. Currently, research is going on semi-organic crystals because of their high damage threshold, wide transparency range, low deliquescence, excellent nonlinear optical efficiency, low angular sensitivity and exceptional mechanical properties [5-8]. Nowadays, borate crystals play a vital role in preparing highly efficient NLO crystals [9, 10]. The NLO materials used for electro-modulation devices, optical parametric oscillators, optoelectronic devices, high-density optical data storage, underwater communications, stereolithography etc., $\mathrm{C}_{8} \mathrm{~B}_{3} \mathrm{O}_{5}$ (CBO) [11], $\mathrm{C}_{5} \mathrm{LiB}_{6} \mathrm{O}_{10}$ $(\mathrm{CLBO}) \quad[12], \quad \mathrm{Sr}_{2} \mathrm{~B}_{2} \mathrm{Be}_{2} \mathrm{O}_{7} \quad$ (SBBO) [13], $\mathrm{K}_{2} \mathrm{AL}_{2} \mathrm{~B}_{2} \mathrm{O}_{7}$ (KABO) [14], etc., are the materials containing borate groups crystals. Several

*E-mail: balad67@ rediffmail.com researchers focus on crystalline phthalic acid complexes and study their linear and nonlinear optical properties [15]. Hence, the new phthalate-based alkali metal complex - crystal of potassiumboro-phthalate - has been grown by slow solvent evaporation technique at room temperature and its optical, electrical and mechanical characteristics have been studied and reported.

\section{Material synthesis}

Potassium-boro-phthalate (KBP) compound was synthesized using high (99\%) purity Merck potassium acid phthalate and boric acid taken in 1:1 molar ratio and dissolving them in deionized water of resistivity $18.2 \mathrm{M} \Omega / \mathrm{cm}$. The mixture was stirred at temperature above $50{ }^{\circ} \mathrm{C}$ and below $60{ }^{\circ} \mathrm{C}$ with the help of magnetic stirrer for more than 4 hours. The prepared solution was allowed to dry at room temperature. The potassium-borophthalate (KBP) compound was obtained by the following chemical reaction: 


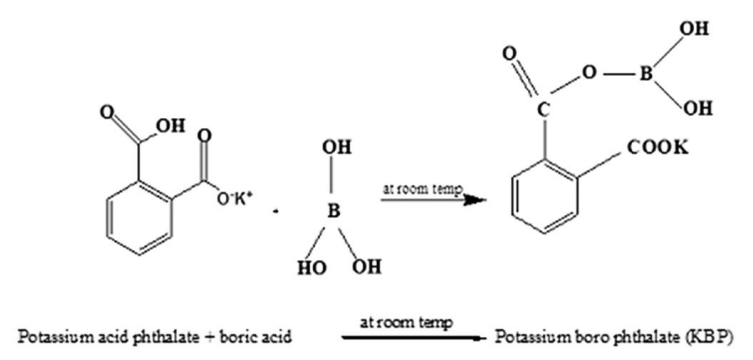

Scheme 1. Reaction scheme for potassium-borophthalate.

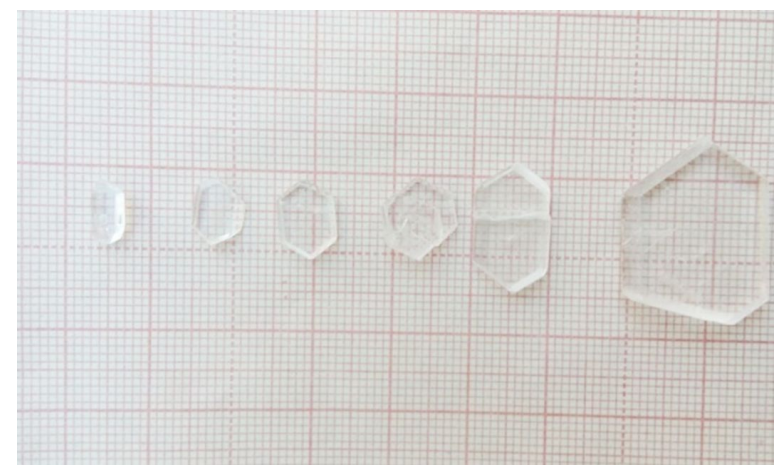

Fig. 1. As-grown bulk crystals of KBP.

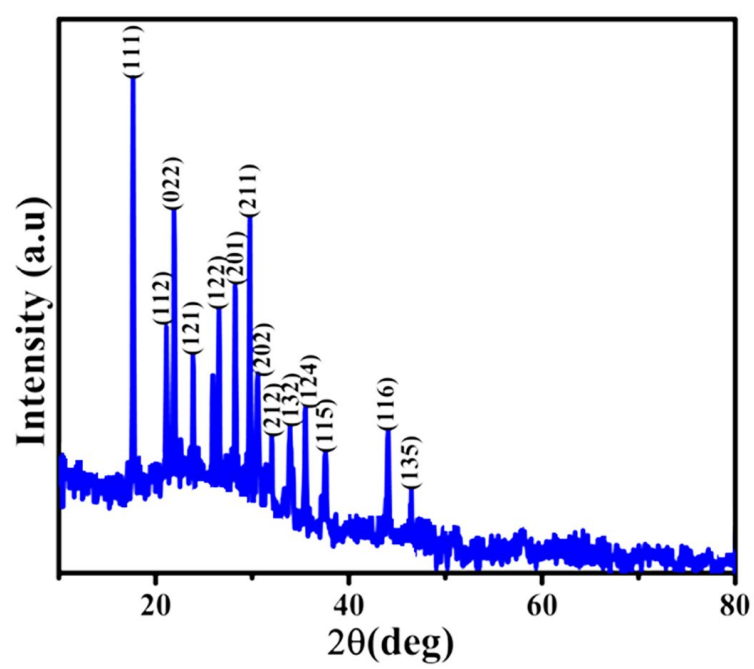

Fig. 2. Powder XRD spectrum of KBP crystal.

\section{Results and discussion}

\subsection{Powder X-ray diffraction studies}

The powder sample of KBP crystal was subjected to powder X-ray diffraction analysis using a Rich Seifert diffractometer with $\mathrm{CuK} \alpha$ $(\lambda=1.5418 \AA)$ radiation. The sample was scanned over the range of $10^{\circ}$ to $80^{\circ}$ at a scan rate of $2 \% \mathrm{~min}$. The recorded X-ray pattern of KBP is shown in Fig. 2. The figure shows sharp and intense peaks which confirm the good crystalline nature of the grown crystal. The peaks have been indexed using a DICVO91 program. 
3.2. Single crystal $X$-ray diffraction studies

The KBP single crystal was subjected to single crystal X-ray diffraction analysis using an ENRAFNONIUS CAD-4 single crystal X-ray diffractometer with $\operatorname{MoK} \alpha(\lambda=0.7170 \AA)$ radiation. The lattice parameters are given in Table 1 . The XRD data reveal that the KBP crystallizes in orthorhombic structure with space group PCA21.

\subsection{Fourier transform infrared spec- troscopy analysis}

FT-IR spectral analysis is one of the techniques to confirm the presence of functional groups in the grown single crystal. Bruker IFS66V spectrometer using $\mathrm{KBr}$ pellet technique was used to record the infrared spectrum of the grown KBP crystal in the wavenumber region of $4000 \mathrm{~cm}^{-1}$ to $400 \mathrm{~cm}^{-1}$. The recorded infrared spectrum is shown in Fig. 3.

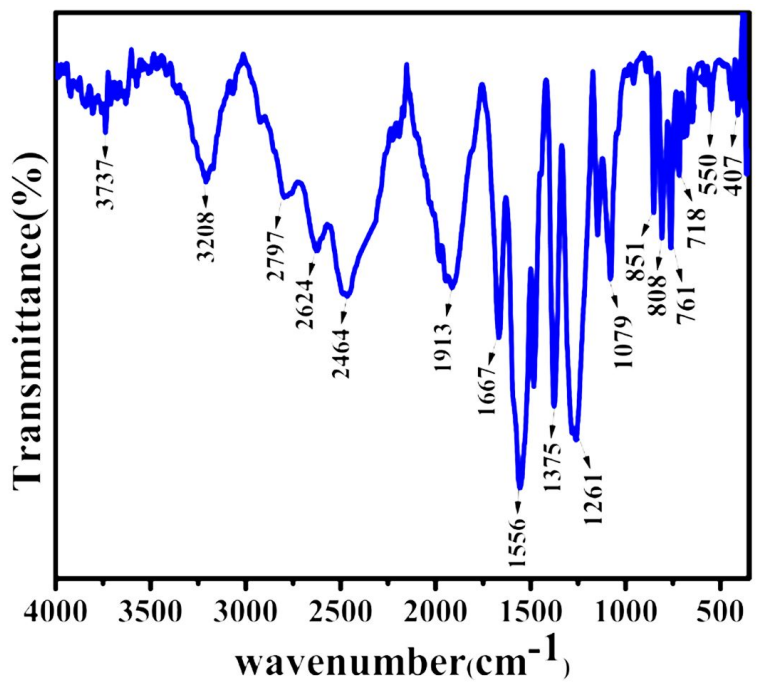

Fig. 3. FT-IR spectrum of KBP crystal.

The characteristic peaks corresponding to molecular stretching, bending and vibrating modes are observed in the FT-IR spectrum.

The presence of various functional groups is summarized in Table 2. A broad peak in the region of $3800 \mathrm{~cm}^{-1}$ to $3200 \mathrm{~cm}^{-1}$ in the spectrum confirms the presence of $\mathrm{O}-\mathrm{H}$ free hydroxyl stretching. The peak observed at $2797 \mathrm{~cm}^{-1}$ has been assigned to $\mathrm{C}-\mathrm{H}$ stretching. The $\mathrm{H}-\mathrm{C}=\mathrm{O}$ stretching appears at $2624 \mathrm{~cm}^{-1}$ and $2464 \mathrm{~cm}^{-1}$. The carbonyl functional group appears at $1913 \mathrm{~cm}^{-1}$. A peak at $1667 \mathrm{~cm}^{-1}$ corresponds to $\mathrm{C}=\mathrm{O}$ stretching. The aromatic ring of $\mathrm{C}-\mathrm{C}$ stretching is observed at $1556 \mathrm{~cm}^{-1}$. The $\mathrm{C}-\mathrm{H}$ rocking gives rise to the peak at $1375 \mathrm{~cm}^{-1}$. The B-O asymmetric and symmetric stretching develops the peaks at $1079 \mathrm{~cm}^{-1}$ and $761 \mathrm{~cm}^{-1}$, respectively. Hence, the presence of boron has been confirmed. The peaks observed at $761 \mathrm{~cm}^{-1}$ and $718 \mathrm{~cm}^{-1}$ are assigned to $=\mathrm{C}-\mathrm{H}$ bending. The incorporation of a metal ion in the grown crystal has been identified from the peaks $550 \mathrm{~cm}^{-1}$ and $407 \mathrm{~cm}^{-1}$.

\subsection{Linear optical properties}

The KBP single crystal of $3 \mathrm{~mm}$ thick sample was placed in the Varian Cary 5E UV-Vis-NIR spectrophotometer. The absorption spectrum was recorded from $190 \mathrm{~nm}$ to $900 \mathrm{~nm}$. The recorded spectrum is shown in Fig. 4. The small absorption in the near UV region is due to weak $\sigma \rightarrow \pi^{*}$ transitions of electrons. As there is no significant absorption in the entire UV-Vis-NIR range with a lower cutoff at $297 \mathrm{~nm}$, the transmission window of the grown crystal satisfies the requirement of electrooptic applications.

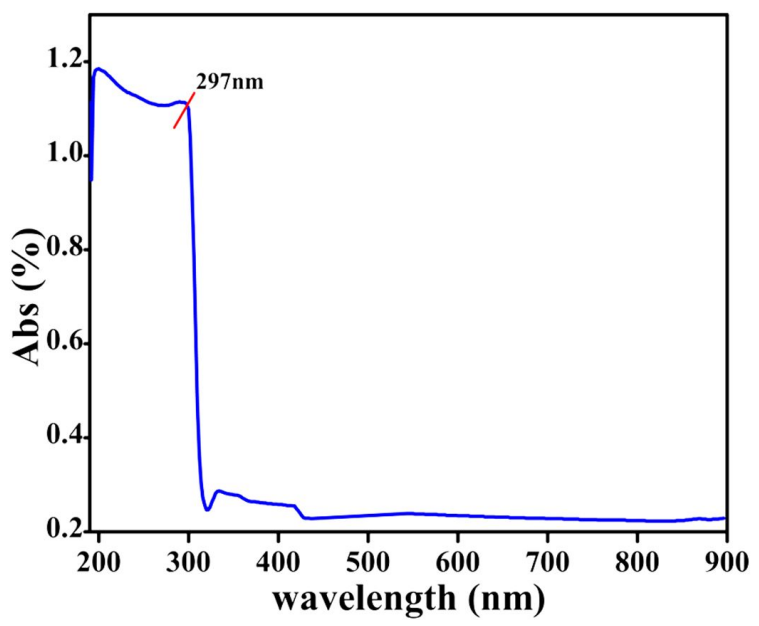

Fig. 4. Linear optical spectrum of KBP crystal.

\subsection{Dielectric analysis}

The dielectric analysis of the grown crystal was carried out using HIOKI 3532-50 LCR HiTESTER 
Table 1. Single crystal XRD data of KBP crystal.

\begin{tabular}{cccccccc}
\hline Sample & \multicolumn{7}{c}{ Lattice parameters } \\
\hline \multirow{2}{*}{$\mathrm{KBP}$} & $\mathrm{a}[\AA]$ & $\mathrm{b}[\AA]$ & $\mathrm{c}[\AA]$ & $\alpha\left[^{\circ}\right]$ & $\beta\left[{ }^{\circ}\right]$ & $\gamma\left[^{\circ}\right]$ & $\mathrm{V}\left[\AA^{3}\right]$ \\
\cline { 2 - 8 } & 6.49 & 9.60 & 13.31 & 90 & 90 & 90 & 829 \\
\hline
\end{tabular}

Table 2. FT-IR assignment for KBP crystal.

\begin{tabular}{cc}
\hline Wavenumber $\left[\mathrm{cm}^{-1}\right]$ & Assignment of various functional groups \\
\hline \hline 3737 & O-H stretching, free hydroxyl \\
3208 & O-H stretching \\
$2797,2624,2464$ & H-C $=$ O stretching \\
1913,1667 & $\mathrm{C}=$ O stretching \\
1556 & C-C stretching (in-ring) \\
1375 & C-H rock \\
1261 & C-O stretching \\
1079 & B-O asymmetric stretching [15] \\
761 & B-O asymmetric stretching [15] \\
718 & $=\mathrm{C}-\mathrm{H}$ bending \\
550,407 & metal ions [16] \\
\hline
\end{tabular}

instrument. The capacitance values of the grown crystal were determined in the frequency range of $50 \mathrm{~Hz}$ to $5 \mathrm{MHz}$ at various temperatures $40{ }^{\circ} \mathrm{C}$, $50{ }^{\circ} \mathrm{C}, 100{ }^{\circ} \mathrm{C}, 150{ }^{\circ} \mathrm{C}, 200{ }^{\circ} \mathrm{C}$.

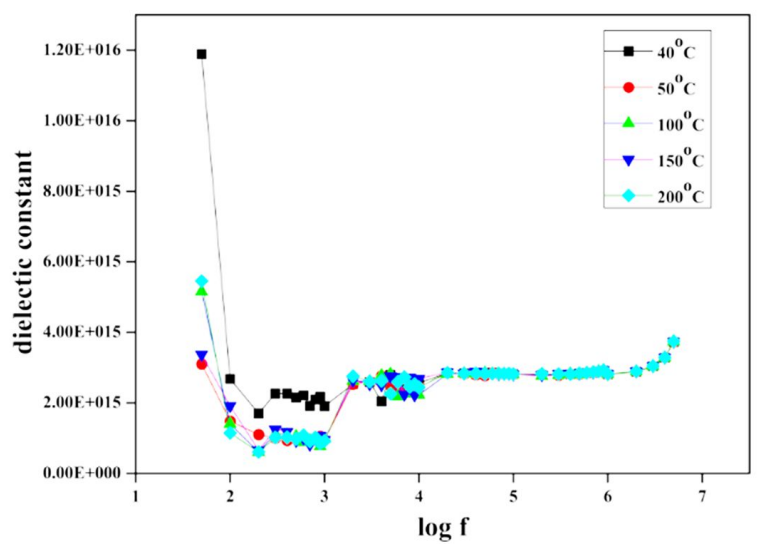

Fig. 5. Dielectric constant of KBP versus log frequency.

The dielectric constant of the grown crystal has been calculated using the formula:

$$
\varepsilon=C t / A \varepsilon_{0}
$$

where $\mathrm{C}$ is the parallel capacitance, $\mathrm{t}$ is thickness of the crystal, and $\epsilon_{0}$ is permittivity of free space.

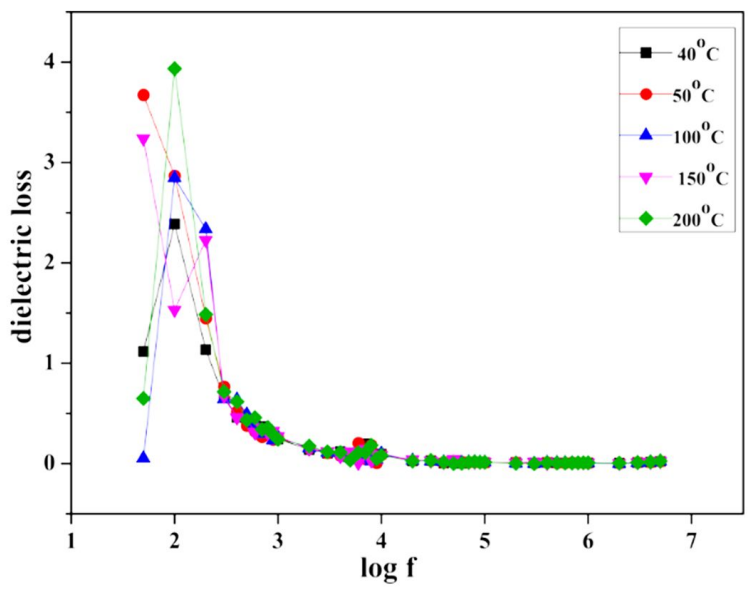

Fig. 6. Dielectric loss of KBP versus log frequency.

Fig. 5 shows the variation of dielectric constant with logarithmic frequency. The dielectric constant has a higher value at low-frequency range which is due to space charge polarization. The value of dielectric constant decreases as the frequency increases which is due to a loss of polarization at high frequency. Fig. 6 shows the variation of dielectric loss with logarithmic frequency. The loss tangent has high value at low frequency and its value 
decreases as frequency increases. The lower value of dielectric loss at higher frequencies reveals that the grown crystals are defect free and have a good optical quality.

\subsection{Vickers microhardness test}

Polished KBP single crystal was subjected to microhardness testing with HEICHRT MD 4000E ULTRA tester to analyze its mechanical behavior. Different loads ranging from $25 \mathrm{~g}$ to $100 \mathrm{~g}$ have been applied in equal steps. The Vickers microhardness number $(\mathrm{Hv})$ values have been calculated for different loads using the relation:

$$
H_{V}=1.8544\left(p / d^{2}\right) k g / m^{2}
$$

where $\mathrm{H}_{\mathrm{V}}$ is Vickers microhardness number in $\mathrm{kg} / \mathrm{mm}^{2}, 1.8544$ is a constant geometrical factor for diamond pyramid, $\mathrm{P}$ is applied load in $\mathrm{g}$, $\mathrm{d}$ is diagonal length of indentation impression in $\mathrm{mm}$.

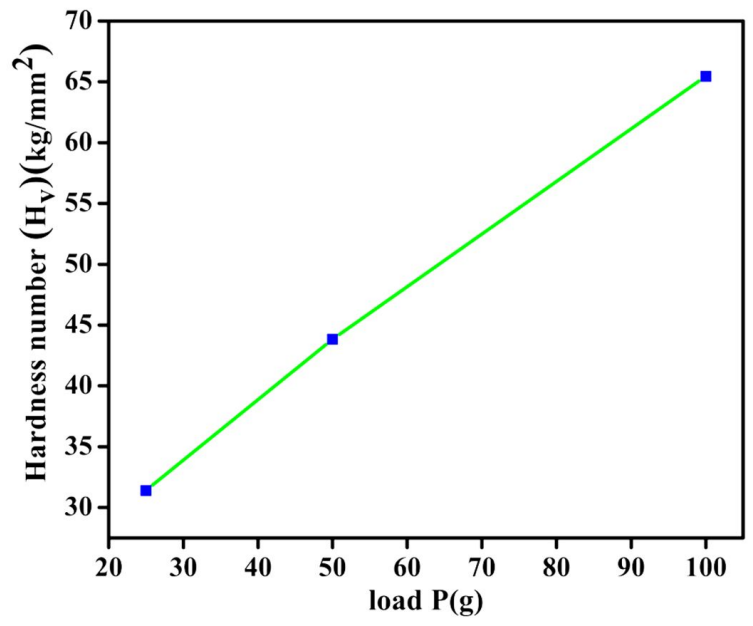

Fig. 7. Hardness number $\left(\mathrm{H}_{\mathrm{V}}\right)$ vs. load $(\mathrm{P})$ for $\mathrm{KBP}$ crystal.

Fig. 7 shows applied load (P) vs. Vickers hardness number (Hv). From the graph, it is noted that the hardness number increases with increasing applied load without any crack in the crystal. This shows that the grown KBP single crystal has good mechanical behavior which is suitable for device fabrication.

\subsection{Nonlinear optical properties}

Second harmonic generation efficiency (SHG) of the grown KBP single crystal was studied using Kurtz and Perry powder technique. The finally powdered crystalline sample of KBP was illuminated with Nd:YAG laser Quanta-Ray of $1064 \mathrm{~nm}$ with the pulse width of $8 \mathrm{~ns}$ and repetition rate of $10 \mathrm{~Hz}$. The potassium-dihydrogen-phthalate (KDP) sample was used as the reference material. The second harmonic signal generated by the crystalline sample of KBP was confirmed by the emission of green radiation $(\lambda=532 \mathrm{~nm})$. A second harmonic signal of $20.8 \mathrm{~mJ}$ was obtained, while the standard KDP crystal gave a SHG signal of $38.7 \mathrm{~mJ}$ for the same input energy. Thus, the SHG efficiency of the grown crystal was found to be equal to approximately 0.5 times that of the KDP. In the employed powder sample, the small crystallites were oriented in different directions. The efficiency of the frequency conversion varied with the particle size and orientation of the crystallites in the capillary tube. Hence, the higher efficiency may be expected to be achieved with the grown crystals of KBP by optimizing the particle size and phase matching angle.

\section{Conclusions}

A semi-organic compound of potassium-borophthalate has been synthesized from potassiumhydrogen-phthalate and boric acid. Optically good quality single crystals of potassium-boro-phthalate (KBP) were successfully grown from the synthesized compound by solution growth technique. Good crystalline nature of the grown crystal was confirmed by the powder XRD analysis. Single crystal XRD analysis revealed that the grown crystal belongs to an orthorhombic crystal system with PCA21 space group. The presence of functional groups was confirmed by FT-IR spectral analysis. UV-Vis-NIR absorption study showed that the grown crystal is transparent in the entire visible and infrared region with the cut-off wavelength of $297 \mathrm{~nm}$ which satisfies the requirements of electrooptic applications. Dielectric studies proved that the KBP single crystals have been grown without 
any defects. On the basis of the Vickers microhardness test, the grown KBP single crystal was found to have good mechanical properties required for device fabrication. The SHG efficiency of KBP was found to be 0.5 times that of the standard KDP crystal.

\section{References}

[1] Kuppers H., Takusagawa F., Koetzle T.F., $J$. Chem. Phys., 82 (1985), 5636.

[2] Karnal A.K., Sexena A., Bhat H.L., Wadhawan V.K., NATHAN T.P.S., J. Cryst. Growth, 289 (2006), 617.

[3] Clegg W., Russo L., Crystal Growth Des., 9 (2009), 1158.

[4] RetajCZAK H., Book of Abstract, Krakow, 2000, p. 21.

[5] Xing G., Jiang., Shao Z., Xu D., ChIn J., Lasers, 14 (1987), 357.

[6] Velsko S., Laser Program Annual Report, Lawrence UCRL- JC 105000, Lawrence Livermore National Laboratory, Livermore Ca., 1990.

[7] Sonal D., Gupta Ranjith S., Pardhan A., Mareano O., Noureddine Melikechi., Desai C.F., $J$. Appl. Phys., 91(2002), 3125.
[8] Rajesh N.P, Kannan V., Ashok M., Sivaji K. Santhana Ragavan P., Ramasamy P., J. Cryst. Growth, 262 (2004), 561.

[9] Chen C.T., Wu B.C., Jiang A.D., You G.M., Sci. Sinica B, 28 (1983), 235.

[10] Chen C.T., Wu Y.C., Jiang A.D., Wu B.C., You G.M., LI R.K., Lin S.J., J. Opt. Soc. Am. B, 6 (1989), 616.

[11] Chang F., Fu P.Z., Wu Y.C., Chen G.J., Xu Z.J., Chen C.T., J. Cryst. Growth, 277 (2005), 289.

[12] Sakai T., Mori Y., Yoshimurai M., Opt. Mater., 23 (2003), 343.

[13] Chang C.T., Wang Y.B., Wu B.C., Wu K.C., Zeng W.L., YU L.H., Nature, 373 (1995), 322.

[14] Hu Z.G., Higashiyama T., Yoshimara M., YaP Y.K., Mori Y., Sasaki T., Jpn. J. Appl. Phys., 37 (1998), L1093.

[15] Sivakumar B., Gokul Raj S., Ramesh KuMar G., Mohan R., J. Crystall. Proc. Tech., 2 (2012), 130.

[16] Chithambaram V., Jeromedas S., ArivuDAINAMBi R., KRISHNAN S., Opt. Laser Technol., 43 (2011), 1229.

Received 2016-10-26 Accepted 2018-04-23 